\title{
Uso de antimicrobianos em pacientes oncológicos em cuidados paliativos
}

\author{
Use of antimicrobials in cancer patients in palliative care
}

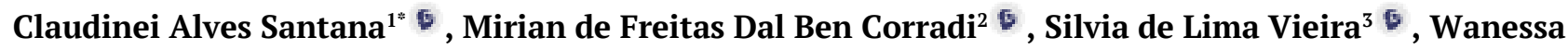 \\ Cassemiro Fernandes ${ }^{4}$
}

\begin{abstract}
${ }^{1}$ Docente do Centro Universitário Senac, São Paulo, São Paulo, Brasil. ${ }^{2}$ Serviço de Controle de Infecção, Hospitalar Hospital SírioLibanês, São Paulo, São Paulo, Brasil. ${ }^{3}$ Gerente de Enfermagem, Hospital Sírio-Libanês, São Paulo, São Paulo, Brasil. ${ }^{4}$ Coordenadora de Curso Instituto de Ensino e Pesquisa, Hospital Sírio-Libanês, São Paulo, São Paulo, Brasil. Autor para correspondência. E-mail: claudinei.santana@ig.com.br
\end{abstract}

\begin{abstract}
Resumo: Introdução: O câncer é caracterizado pela proliferação celular desordenada de origem genética sendo durante muitos séculos um desafio para medicina. As abordagens terapêuticas a depender das reservas fisiológicas e tipo de câncer que acomete o paciente podem comprometer sistemas orgânicos importantes, sendo que parte dos pacientes não respondem adequadamente ao tratamento dando início ao cuidado paliativo. $\mathrm{O}$ uso de antimicrobianos nesta fase é questionável devido aos reais benefícios aos pacientes quando se considera unicamente o aumento da sobrevida. Entretanto, as evidências apontam para o uso dos antimicrobianos com o objetivo de melhorar a qualidade de vida dos pacientes, com controle de sintomas e promoção de conforto. Objetivo deste trabalho foi realizar revisão sistemática da literatura sobre o uso de antimicrobianos em pacientes com câncer em cuidados paliativos e suas reações adversas e benefícios para conforto e tratamento de sintomas. Revisão e discussão: Estudo de revisão da literatura internacional em bases de dados internacionais (PubMed e ScienceDirect) por 2 pesquisadores independentes com proposta de abordar com profundidade o tema e responder ao objetivo proposto. A partir da metodologia adotada formam selecionados 23 artigos para a elaboração do artigo de revisão e as reações adversas são relatadas em estudos relacionados ao uso de antimicrobianos em pacientes oncológicos, porém, não foram descritos como sendo o objetivo principal dos estudos e do ponto de vista de promoção de conforto e controle de sintomas o uso de antimicrobianos nos estudos apresentaram resultados favoráveis, porém, com respostas subjetivas que evidenciam a necessidade de protocolos e score de avaliação. Considerações finais: Os pacientes em cuidado paliativo e uso de antimicrobianos possuem dilemas na gestão do cuidado no fim de vida importantes que necessitam ser avaliados com profundidade em relação a benefícios do uso e presença de reações adversas que possam causar prejuízos nesse momento crítico.
\end{abstract}

Palavras-chave: antibiótico, antimicrobiano, câncer, infecção, cuidado paliativo, reação adversa.

Abstract: Introduction: Cancer is characterized by disorderly cell proliferation of genetic origin and has been a challenge for medicine for many centuries. Therapeutic approaches depending on the physiological reserves and the type of cancer that affects the patient can compromise important organic systems, and some patients do not respond adequately to the treatment, initiating palliative care. The use of antimicrobials at this stage is questionable due to the real benefits to patients when considering only increased survival. However, the evidence points to the use of antimicrobials in order to improve the quality of life of patients, with symptom control and the promotion of comfort. Objective of this work was to carry out a systematic review of the literature on the use of antimicrobials in cancer patients in palliative care and its adverse reactions and benefits for comfort and treatment of symptoms. Review and discussion: International literature review study in international databases (PubMed and ScienceDirect) by 2 independent researchers with the proposal to approach the theme in depth and respond to the proposed objective. From the adopted methodology, 23 articles were selected for the preparation of the review article and adverse reactions are reported in studies related to the use of antimicrobials in cancer patients, however, they were not described as the main objective of the studies and from the point of view of promoting comfort and symptom control the use of antimicrobials in the studies showed favorable results, however, with subjective responses that demonstrate the need for protocols and evaluation scores. Final considerations: Patients in palliative care and use of antimicrobials have important dilemmas in the management of end-of-life care that need to be thoroughly evaluated in relation to the benefits of the use and the presence of adverse reactions that can cause losses at this critical moment.

Keywords: antibiotic, antimicrobial, cancer, infection, palliative care, adverse reaction. 


\section{Introdução}

O câncer é caracterizado pela proliferação celular desordenada de origem genética de apresentação diversificada sendo durante muitos séculos um desafio para medicina com impacto na área da saúde (Hassanpour \& Dehghani, 2017)

Em 2018, as estimativas de casos de câncer no mundo foram de 17 milhões com 9.6 milhões de óbitos (https://www.cancerresearchuk.org/health-professional/cancer-statistics/worldwide-cancer). No Brasil dados do Instituto Nacional do Câncer (INCA) estimam a ocorrência de 600 mil novos casos de câncer ao ano para o para o biênio 2018 - 2019 (https://www.inca.gov.br/numeros-de-cancer).

O desfecho do câncer é variável. Para alguns tipos de câncer, atingem-se altas taxas de cura, como em pacientes infantis com leucemia. Em outras neoplasias, não são possíveis índices elevados de cura e a mortalidade em decorrência da neoplasia é importante, como, por exemplo, em melanomas (Leonardi et al., 2018; Zwaan et al., 2015). Considerando-se que os principais objetivos do tratamento do câncer são aumento da sobrevida e da qualidade de vida, são muito utilizados os conceitos de sobrevida global, sobrevida em determinado tempo (6 meses, 1 ano, 5 anos, etc.), sobrevida livre de progressão de doença e tempo para progressão (Machado et al., 2010).

$\mathrm{O}$ tratamento do câncer requer uma abordagem multiprofissional com modalidades variadas como cirurgia, quimioterapia, radioterapia, imunoterapia, hormonioterapia (Abotaled et al., 2018).

As abordagens terapêuticas, a depender das reservas fisiológicas e tipo de câncer que acomete o paciente, podem comprometer sistemas orgânicos importantes, sendo necessário a aplicação de escalas de performance e seguimento regular por equipe multiprofissional com intuito de diminuir morbidades relacionadas ao tratamento (Berkey, 2010; Sarfati, Koczwara, \& Jackson, 2016; Kroschinsky et al., 2017).

No entanto, uma parcela dos pacientes oncológicos não responde adequadamente as modalidades de tratamento, ocasionando a progressão da doença sem expectativa de cura e direcionando o paciente ao cuidado paliativo para fim de vida (Sarfati, Koczwara, \& Jackson, 2016; Kroschinsky et al., 2017; Berkey, 2010).

Segundo a Organização Mundial de Saúde (OMS), os cuidados paliativos foram definidos como uma abordagem que melhora a qualidade de vida dos pacientes e suas famílias diante dos problemas associados a doenças com risco de vida, através da prevenção e alívio do sofrimento por meio da identificação precoce e avaliação adequada e tratamento da dor e outros problemas, físicos, psicossociais e espirituais (https://www.who.int/cancer/palliative/definition/en/). Diante deste contexto, o cuidado paliativo propõe cuidados de conforto, cuidados de suporte e gerenciamento de sintomas para manter a melhor qualidade possível de vida nesta etapa final, buscando alinhar os cuidados e intervenções terapêuticas com as expectativas do paciente (https://medlineplus.gov/ency/patientinstructions/000536.htm).

Aproximadamente $90 \%$ dos pacientes hospitalizados com câncer avançado são tratados com antimicrobianos durante a semana anterior ao óbito (Thompson et al., 2012; Juthani-Mehta, Malani, \& Mitchell, 2015). Com o avanço da doença, fragilidade do sistema imunológico induzida pelos tratamentos e pelo próprio câncer e com os inúmeros procedimentos invasivos a que são submetidos, muitos pacientes com câncer na etapa final da doença apresentam infecções. Estas acometem as mais variadas topografias: corrente sanguínea, infecções intra-abdominais e do sistema urinário, infecções respiratórias e orofaringe, pele e partes moles que favorecem a piora do quadro clínico e da qualidade de vida dos doentes (Pereira, Watanabe, \& Wolch., 1998; White et al., 2003; Thompson et al., 2012; Mohammed et al., 2014; Ramada \& Feijó Barroso, 2016; Macedo et al., 2018).

O uso de antimicrobianos nesta fase é questionável devido aos reais benefícios aos pacientes quando se considera unicamente o aumento da sobrevida. Entretanto, as evidências apontam para o uso dos antimicrobianos com o objetivo de melhorar a qualidade de vida dos pacientes no controle de sintomas e promoção de conforto (Nakagawa et al., 2010; Stiel et al., 2012; Rosenberg et al., 2013; Furuno et al., 2014; Mohammed et al., 2014; Merel et al., 2016; Macedo et al., 2018).

Em um estudo de revisão foram observadas taxas de 19 a $84 \%$ de uso de antimicrobianos em pacientes oncológicos para controle de sintomas (Rosenberg et al., 2013). Dentre os pacientes com câncer hematológico, 90\% receberam antibióticos na última semana de vida (Juthani-Mehta \& Allore, 2019). Entretanto, ambos os estudos encontraram muitos casos nos quais não havia evidencias clínicas para a prescrição e utilização dos antimicrobianos (Rosenberg et al., 2013; Juthani-Mehta \& Allore, 2019).

Estudo realizado pelo Instituto Nacional do Câncer avaliando 870 pacientes oncológicos em unidade de cuidados paliativos observou uso de antimicrobianos em $74 \%$ dos pacientes, sendo $48 \%$ destes sem citação de diagnóstico de doença infecciosa (Ramada \& Feijó Barroso, 2017). 
O uso de antimicrobianos pode levar à ocorrência de reações adversas sendo definida pela Organização Mundial de Saúde como qualquer resposta prejudicial ou indesejável, não intencional, a um medicamento, que ocorre nas doses usualmente empregadas para profilaxia, diagnóstico ou terapia de doenças (WHO, 2002).

Em relação as reações adversas relacionadas aos antimicrobianos importante ressaltar as toxicidades hematológicas, hepáticas, renais, gastrointestinais e neurológicas com potencial de mortalidade (Yilmaz \& Özcengiz, 2017).

Em pacientes oncológicos, já debilitados pelo tratamento e em cuidados paliativos, há necessidade de se avaliar os reais benefícios do uso dos antimicrobianos. Nestes pacientes já fragilizados pela própria doença, pode haver piora do quadro a partir das reações adversas (Pereira, Watanabe, \& Wolch, 1998; White et al., 2003).

O farmacêutico clinico tem papel importante atuando em conjunto com a equipe multiprofissional no acompanhamento e avaliação das ações em saúde ao paciente oncológico (Brasil, 2012).

A utilização de antimicrobianos em cuidados paliativos em pacientes oncológicos exige avaliação pela equipe multiprofissional que realiza o acompanhamento clínico do paciente com análise dos seus riscos e benefícios.

Este artigo de tem como objetivo realizar revisão sistemática da literatura sobre o uso de antimicrobianos em pacientes com câncer em cuidados paliativos, e descrever os riscos de reações adversas aos fármacos antimicrobianos em pacientes com câncer em cuidado paliativo, e as evidências existentes na literatura dos benefícios do uso para conforto e controle de sintomas em pacientes com câncer em cuidado paliativo.

\section{Revisão}

Trata-se de uma revisão ampla da literatura realizada por 2 pesquisadores independentes com proposta de abordar com profundidade o tema e responder ao objetivo proposto.

Foram realizadas buscas por artigos originais, artigos de revisão e artigos de "ponto de vista" de profissionais internacionais na integra no período de março a maio de 2019 em bases de dados internacionais (PubMed e ScienceDirect) com utilização dos termos DeCS/MeSH: antibiotic, antimicrobial, cancer, infection, palliative care, adverse reaction.

Após a identificação dos artigos a partir das palavras chaves, foi realizada leitura do título e resumo, sendo excluídos artigos em que o uso de antimicrobianos não era relacionado a pacientes oncológicos em cuidados paliativos.

A partir da metodologia adotada formam selecionados 23 artigos para elaboração do artigo de revisão apresentados no quadro 1.

A utilização de antimicrobianos em final de vida é uma prática recorrente em hospitais pelo mundo, demonstrando a necessidade de uma avaliação quanto aos benefícios do uso (White et al., 2003; Thompson et al., 2012).

O uso de antimicrobianos em pacientes oncológicos em cuidado paliativo pode ser um dilema quanto a decisão de tratar, manter ou suspender o tratamento antimicrobiano para infecção nestes pacientes (Macedo et al., 2018).

Inúmeros reações adversas podem surgir da administração antimicrobiana em pacientes oncológicos, como desenvolvimento e transmissão de organismos multirresistentes a múltiplos fármacos, interações medicamentosas, reações adversas, toxicidade, infecção por Clostridium difficile e aumento dos custos de cuidados de saúde (Mirhosseini et al., 2006; Juthani-Mehta \& Alorre, 2019).

Existem poucos dados publicados sobre a frequência de reações adversas associadas a antimicrobianos em cuidados paliativos. Estima-se serem altas as frequencias nesta população, dado as conhecidas incidências de reações adversas dos antimicrobiano em populações saudáveis (Furuno, Noble, \& Fromme, 2016).

As reações adversas dos antimicrobianos são, muitas vezes, banalizadas, porém, estão presentes e podem contribuir para a piora do quadro do paciente. No estudo realizado na Universidade de Michigan, à Vancomicina e Piperacilina/tazobactam estavam entre os fármacos mais prescritos (Thompson et al., 2012). Segundo a literatura, a utilização de antimicrobianos está associada a infecção por Clostridium difficile e o uso da Piperacilina/tazobactam esteve associado a diarreia com frequência superior a $10 \%$ dos pacientes em uso (Prabhavathi \& Evan, 2017). A diarreia pode comprometer a qualidade de vida e conforto do paciente e necessitar de mais intervenções pela equipe de saúde.

$\mathrm{O}$ uso do sulfametoxazol/trimetroprim em pacientes oncológicos podem apresentam alterações laboratoriais importantes como agranulocitose, anemia aplástica, eosinofilia, anemia hemolítica, hipoprotrombinemia, leucopenia, anemia megaloblástica, metemoglobinemia, neutropenia, trombocitopenia (Prabhavathi \& Evan, 2017). Tais eventos hematológicos podem favorecer processos infecciosos, necessitando da inclusão de novos antimicrobianos. 
Quadro 1. Distribuição dos artigos segundo título, ano e tipo de estudo.

\begin{tabular}{|c|c|c|}
\hline Título & Ano & Tipo de estudo \\
\hline Design and analysis of longitudinal trials of antimicrobial use at the end of life: to give or not to give? & 2019 & Revisão \\
\hline Why is optimisation of antimicrobial use difficult at the end of life? & 2019 & $\begin{array}{c}\text { Perspectiva } \\
\text { pessoal }\end{array}$ \\
\hline Antimicrobial use in patients at the end of life in an Australian hospital & 2019 & Original \\
\hline Current opinion about antimicrobial therapy in palliative care: an update. & 2019 & Revisão \\
\hline Antimicrobial therapy in palliative care: an overview. & 2018 & Revisão \\
\hline Physician Perceptions Regarding Antimicrobial Use in End-of-Life Care. & 2018 & Original \\
\hline $\begin{array}{c}\text { Antibiotics in palliative care: less can be more. Recognising overuse is easy. The real challenge is } \\
\text { judicious prescribing. }\end{array}$ & 2018 & $\begin{array}{c}\text { Perspectiva } \\
\text { pessoal }\end{array}$ \\
\hline Burden and Management of Multidrug-Resistant Organisms in Palliative Care. & 2017 & Revisão \\
\hline Antimicrobial Use in Patients on a Comfort Care Protocol: A Retrospective Cohort Study & 2016 & Original \\
\hline High Frequency of Antimicrobials Use in Palliative Care: Are We Moving in the Right Direction? & 2016 & $\begin{array}{c}\text { Perspectiva } \\
\text { pessoal }\end{array}$ \\
\hline Should we refrain from antibiotic use in hospice patients? & 2016 & $\begin{array}{c}\text { Perspectiva } \\
\text { pessoal }\end{array}$ \\
\hline $\begin{array}{l}\text { Antibiotic Treatment in End-of-Life Cancer Patients-A Retrospective Observational Study at a Palliative } \\
\text { Care Center in Sweden }\end{array}$ & 2016 & Original \\
\hline $\begin{array}{c}\text { Antimicrobials at the End of Life } \\
\text { An Opportunity to Improve Palliative Care } \\
\text { and Infection Management }\end{array}$ & 2015 & $\begin{array}{l}\text { Perspectiva } \\
\text { pessoal }\end{array}$ \\
\hline The pattern of infection and antibiotics use in terminal cancer patients. & 2014 & Original \\
\hline $\begin{array}{l}\text { Antimicrobial use for symptom management in patients receiving hospice and palliative care: a } \\
\text { systematic review }\end{array}$ & 2013 & Revisão \\
\hline Is it appropriate to withdraw antibiotics in terminal patients with cancer with infection?. & 2013 & Original \\
\hline Antimicrobial use at the end of life among hospitalized patients with advanced cancer & 2012 & Original \\
\hline $\begin{array}{l}\text { Antibiotics in palliative medicine--results from a prospective epidemiological investigation from the } \\
\text { HOPE survey. }\end{array}$ & 2012 & Original \\
\hline $\begin{array}{l}\text { Can anti-infective drugs improve the infection-related symptoms of patients with cancer during the } \\
\text { terminal stages of their lives? }\end{array}$ & 2010 & Original \\
\hline The role of antibiotics in the management of infection-related symptoms in advanced cancer patients. & 2006 & Original \\
\hline Antimicrobial use in patients with advanced cancer receiving hospice care. & 2003 & Original \\
\hline $\begin{array}{l}\text { A retrospective review of the frequency of infections and patterns of antibiotic utilization on a palliative } \\
\text { care unit }\end{array}$ & 1998 & Original \\
\hline
\end{tabular}

Em estudo prospectivo realizado em Taiwan com 378 pacientes com cancer terminal e recebendo antimicrobianos, observou-se que 295 receberam antibióticos à admissão. O uso de antimicrobianos esteve relacionado a aumento na sobrevida quando administrados para os pacientes vivos após uma semana de internação. Entretanto, a introdução de antimicrobianos nos dois dias pré óbito não estiveram relacionados a benefícios em virtude da presença de reações adversas e piora do quadro clinico geral (Chih et al., 2013)

Um ponto importante de reflexão relacionado ao uso irracional de antimicrobianos é a pressão seletiva, levando ao surgimento de cepas resistentes com comprometimento da resposta clínica ao tratamento (https://www.who.int/antimicrobial-resistance/en/). Tais bactérias acabam por comprometer a situação epidemiológica dos serviços de saúde, com a ocorrência de transmissões cruzadas, surtos e comprometimento do tratamento e da qualidade de vida de outros pacientes oncológicos e não oncológicos (Datta \& JuthaniMehta, 2017). Os pacientes colonizados por bactérias multirresistentes podem representar um reservatório deste microrganismo na UTI onde muitos encontram-se em processo de fim de vida (Levin et al., 2010)

Em um estudo para a valiar a percepção de 637 médicos quanto ao uso de antimicrobianos em pacientes terminais, metade dos médicos participantes $(49,8 \%)$ afirmaram que o uso de antimicrobianos no final da vida contribui à seleção de resistência (Gaw et al., 2018).

Os potenciais benefícios dos antimicrobianos são alívio dos sintomas, conforto e aumento da sobrevida (Macedo et al., 2018). Porém, para avaliação dos riscos e benefícios desta dimensão do cuidado, a equipe multiprofissional necessita de experiência para a tomada de decisão.

Estudo realizado por Thompson et al. (2012) encontrou 126 de 145 pacientes recebendo antimicrobianos e $99(78,5 \%)$ pacientes tiveram como principal justificativa de uso o conforto com o controle dos sintomas (Thompson et al., 2012)

A prática de prescrição de antimicrobianos para tratar sintomas parece trazer benefícios para pacientes oncológicos em cuidados paliativos. Nakagawa et al. (2010) avaliaram 71 pacientes em cuidados paliativos que receberam fármacos antimicrobianos. $33,1 \%$ tiveram melhoras dos sintomas, porém, a melhora dos 
sintomas foi dificilmente observada quando o medicamento foi administrado na última semana de vida com diminuição de apenas redução da febre de $28,4 \%$.

A febre não se mostra um sintoma interessante a ser considerado de forma isolada para o início do tratamento com antimicrobianos, pois pode estar presente como manifestação de infecção ou de progressão da doença oncológica (Broom et al., 2019). A resposta geral aos antimicrobianos e o controle dos sintomas das infecções foram observados em apenas $40 \%$ dos 102 pacientes em estudo de revisão retrospectivo no final da década de 90 (Vitetta, Kenner, \& Sali, 2000).

Em outro estudo de revisão da literatura sobre utilização de antimicrobianos avaliando o tratamento de sintomas neste grupo de pacientes, não foi possível concluir os beneficios reais pois os metodos e escalas de avaliação e terminologias utilizados para determinar os beneficios eram vagos e subjetivos (Rosenberg et al., 2013).

No que diz respeito ao tratamento de infecções em pacientes com cuidado paliativo, observa-se um aumento na sobrevida dos pacientes em sepse (média de 15 dias) e dos pacientes com infecções em diversas topografias (48 dias), porém, fatores associados ao paciente que possam levar a esses benefícios não estão claros (Thai et al., 2012).

A avaliação de benefícios no tratamento dos sintomas nas últimas 2 semanas de vida dos pacientes oncológicos também foi objeto de estudo retrospectivo observacional na Suécia. Na revisão de prontuário de 79 pacientes oncológicos em cuidados paliativos, observou-se $37 \%$ dos pacientes nos quais o tratamento resultou em alívio evidente dos sintomas. Quando a indicação do tratamento antibiótico foi para evitar ou tratar a sepse, o alívio dos sintomas foi alcançado em $50 \%$ dos pacientes (Helde-Frankling et al., 2016).

Entretanto, um estudo retrospectivo com pacientes em câncer terminal avaliando a prevalência de infecção, sitios infecciosos e melhora de sintomas pelo uso de antimicrobianos mostrou que, dentre 243 pacientes em uso de antimicrobianos, 42 pacientes (17,3\%) obtiveram melhoras dos sintomas, 71 pacientes $(29,2 \%)$ não obtiveram alteração na sintomatologia e 130 pacientes $(53,5 \%)$ revelaram deterioração dos sintomas (Mohammed et al., 2014).

A escolha por parte do paciente do uso de antimicrobianos em cuidado paliativo é uma realidade que necessita ser observada. Em um estudo com 255 pacientes, 53 pacientes $(20,8 \%)$ solicitaram antimicrobianos como medidas para prolongamento da vida e controle dos sintomas; 123 pacientes $(48,2 \%)$ solicitaram apenas o controle dos sintomas e 79 pacientes $(31,0 \%)$ não solicitaram intervenções que pudessem prolongar a vida principalmente devido à sua condição muito debilitada (White et al., 2003).

Além do desejo do paciente, a interação médico-paciente também pode direcionar o uso de antimicrobianos. Um estudo com 637 médicos entrevistados, concluiu que 86,2\% dos médicos acreditava que é necessário respeitar o desejo do paciente em continuar o uso de antimicrobianos (Gaw et al., 2018). A análise dessa afirmação é importante, pois, o fato do profissional médico acreditar que se deve respeitar a decisão do paciente a continuar com o tratamento antimicrobiano adicionada a sua falta de evidência clínica em suspender o tratamento em final de vida pode ser um fator importante para colaborar com a manutenção da prescrição em pacientes oncológicos.

É muito comum a divergência de opinião entre profissionais médicos em relação à utilização de antimicrobianos no tratamento paliativo dos pacientes sob seus cuidados. Grande é o debate quanto às questões éticas e legais relacionadas à retirada ou manutenção de antimicrobianos frente ao desejo do paciente e familiares em continuar com o tratamento (Macedo et al., 2018).

Em regras gerais, muitos profissionais médicos e familiares consideram os antimicrobianos como um padrão mínimo de tratamento no cuidado terminal, acreditando que os antibióticos são inofensivo e podem melhorar o controle dos sintomas (Lee, 2018).

A futilidade terapêutica é definida como o uso de formas de tratamento cujo efeito é mais nocivo do que os efeitos da própria doença de base, ou cujo efeito é inútil, porque a cura é impossível e o benefício esperado é menor do que os inconvenientes previsíveis (Lima, 2006; Broom et al., 2019). Uma revisão retrospectiva do uso de antibióticos em pacientes que evoluíram ao óbito entre janeiro de 2015 e julho de 2015, observou que 86 de 137 pacientes receberam antimicrobianos no final da vida e que 29 pacientes (33,7\%) receberam antimicrobianos após a futilidade terapêutica ter sido documentada. Isso demostra que o médico ainda tem dificuldades em aceitar o fim de vida e a não intervenção medicamentosa (Dyer et al., 2019).

Outra prática bastante comum é o uso empírico de antimicrobianos em pacientes em cuidados paliativos. O Serviço de Saúde da Universidade de Michigan avaliou 131 pacientes terminais com câncer atendidos em consulta. Encontraram 70 pacientes receberando antimicrobianos, sendo a terapia empírica em 54 pacientes (Thompson et al., 2012). A utilização empirica expõe o paciente paliativo a novos antimicrobianos, ampliando a farmacoterapia do paciente, promovendo o aumento no risco de interações medicamentosas que podem ser prejudiciais ao paciente e selecionando flora multirresistente. 


\section{Discussão}

O uso de antimicrobianos em pacientes oncológicos em cuidados paliativos se apresenta como um desafio à todos os profissionais da equipe multiprofissinal envolvidos no cuidado.

É uma realidade em que o objetivo do uso ainda não é claro, necessitando de reflexões da equipe multiprofissional quanto aos reais benefícios desejados e os benefícios efetivamente alcançados a estes pacientes. Além disto, é essencial que sejam alinhadas as expectativas do paciente e da equipe multiprofissional em relação aos cuidados.

As reações adversas são relatadas em estudos relacionados ao uso de antimicrobianos em pacientes oncológicos, porém, não são avaliados quanto a contribuição para piora do quadro de saúde do paciente (Mirhosseini et al., 2006; Juthani-Mehta \& Allore, 2019). Nos estudos em que havia descrição das reações adversas, não encontramos correlação com dose do antimicrobiano ou vias de administração e, tão pouco, encontramos descrição dos protocolos de quimioterapia a que estes pacientes foram submetidos. Tais informações poderiam em segunda estância, fomentar uma análise mais profunda de possíveis interações entre as classes farmacológicas e aprimorar o conhecimento sobre as reações adversas presents (Mirhosseini et al., 2006; Thompson et al., 2012; Furano et al., 2016; Juthani-Mehta \& Allore, 2019).

É possível refletir, também, sobre a real importância clinica das reações adversas aos pacientes oncológicos em final de vida. Diante da gravidade da doença e priorização da manutenção da vida por parte dos médicos, a presença das reações adversas talvez não seja o principal fator de risco a saúde do paciente na avaliação desse profissional. Isto pode justificar o fato das reações adversas não serem o objetivo principal de estudo dos artigos. Entretanto, num contexto de cuidados paliativos, a priorização do conforto como mitigação dos eventos adversos talvez devesse ser o principal objetivo a ser observado na escolha da modalidade terapêutica.

Do ponto de vista de promoção de conforto e controle de sintomas, o uso de antimicrobianos nos estudos apresentaram resultados favoráveis e reforçam essa prática proporcionado qualidade de vida aos pacientes e seus familiars (Vitetta, Kenner, \& Sali., 2000; Nakagawa et al., 2010; Helde-Frankling et al., 2016). No entanto, há necessidade da elaboração de protocolos ou "scores" para avaliar objetivamente a melhora do conforto do paciente e sintomas já que são por muitas vezes subjetivos sem medotologias claras de avaliação (Thai et al., 2012; Rosenber et al., 2013).

Outro ponto relevante seria a elaboração de protocolos ou scores para avaliar a indicação de utilização dos antimicrobianos com este objetivo e o tempo de utilização dos antimicrobianos, tendo em vista que estudos demonstram que não houve melhora de sintomas quando foram introduzidos nas 24 - 48 horas que antecederam o óbito, demonstrando a necessidade de critérios para avaliar qual paciente realmente se beneficiaria desse tratamento (Chih et al., 2013).

Os estudos mostram o desejo do paciente em utilizar antimicrobianos no fim de vida para finalidade de conforto e o interesse dos médicos em respeitar essa decisão. Porém, a ausência de evidencias clinicas precisas de conforto e melhora dos sintomas podem acarretar intensas crises éticas e morais para equipe de saúde quanto a manter o uso do antimicrobiano (White et al., 2003; Gaw et al., 2018).

É importante ressaltar a presença frequente de prescrição de antimicrobianos na ausência de sinais especificos de processos infecciosos nos estudos relatados (Broom et al., 2019), e o impacto que essa prática pode proporcionar no surgimento de cepas bacterianas resistentes no ambiente hospitalar (Levin et al., 2010; Datta \& Juthani-Mehta, 2017).

É notória a dificuldade em se obter uma conclusão entre o uso de antimicrobianos em cuidados paliativos em pacientes oncológicos, pois, o desafio é equilibrar o cuidado compassivo com os pacientes que sofrem de câncer em cuidados paliativos (Macedo \& Bonito, 2019).

\section{Considerações finais}

Os pacientes em cuidado paliativo e uso de antimicrobianos possuem dilemas na gestão do cuidado no fim de vida importantes que necessitam ser avaliados com profundidade em relação a benefícios do uso e presença de reações adversas que possam causar prejuízos nesse momento crítico.

Cabe ressaltar a necessidade da atuação da equipe multiprofissional nas ações de saúde ao paciente oncológico em cuidado paliativo em uso de antimicrobianos para aprimoramento do conhecimento e manejo do cuidado. 


\section{References}

Abotaleb, M., Kubatka, P., Caprnda, M., Varghese, E., Zolakova, B., Zubor, P., ... \& Büsselberg, D. 2018. Chemotherapeutic agents for the treatment of metastatic breast cancer: An update. Biomedicine \& Pharmacotherapy, 101, 458-477.

Berkey, F. J. 2010. Managing the adverse effects of radiation therapy. American Family Physician, 82(4), 381-388.

Brasil. 2012. Resolução n 565 de 6 de dezembro de 2012. Ementa: Dá nova redação aos artigos $1^{\circ}, 2^{\circ}$ e $3^{\circ}$ da Resolução/CFF no 288 de 21 de março de 1996. Diário oficial da união. 07 dez 2012. Recuperado de http://www.cff.org.br/userfiles/file/resolucoes/565.pdf

Broom, J., Broom, A., Good, P., \& Lwin, Z. 2019. Why is optimisation of antimicrobial use difficult at the end of life?. Internal Medicine Journal, 49(2), 269-271.

Chih, A. H., Lee, L. T., Cheng, S. Y., Yao, C. A., Hu, W. Y., Chen, C. Y., \& Chiu, T. Y. 2013. Is it appropriate to withdraw antibiotics in terminal patients with cancer with infection?. Journal of Palliative Medicine, 16(11), 1417-1422.

Datta, R., \& Juthani-Mehta, M. 2017. Burden and management of multidrug-resistant organisms in palliative care. Palliative Care: Research and Treatment, 10, 1178224217749233.

Dyer, J., Vaux, L., Broom, A., \& Broom, J. (2019). Antimicrobial use in patients at the end of life in an Australian hospital. Infection, Disease \& Health, 24(2), 92-97.

Furuno, J. P., Noble, B. N., \& Fromme, E. K. (2016). Should we refrain from antibiotic use in hospice patients?. Expert Rev Anti Infect Ther, 14(3), 277-80.

Furuno, J. P., Noble, B. N., Horne, K. N., McGregor, J. C., Elman, M. R., Bearden, D. T., ... \& Fromme, E. K. 2014. Frequency of outpatient antibiotic prescription on discharge to hospice care. Antimicrobial Agents and Chemotherapy, 58(9), 5473-5477.

Gaw, C. E., Hamilton, K. W., Gerber, J. S., \& Szymczak, J. E. 2018. Physician perceptions regarding antimicrobial use in end-of-life care. Infection Control \& Hospital Epidemiology, 39(4), 383-390.

Hassanpour, S. H., \& Dehghani, M. 2017. Review of cancer from perspective of molecular. Journal of Cancer Research and Practice, 4(4), 127-129.

Helde-Frankling, M., Bergqvist, J., Bergman, P., \& Björkhem-Bergman, L. 2016. Antibiotic treatment in endof-life cancer patients-A retrospective observational study at a palliative care center in Sweden. Cancers, $8(9), 84$.

Juthani-Mehta, M., \& Allore, H. G. (2019). Design and analysis of longitudinal trials of antimicrobial use at the end of life: to give or not to give?. Therapeutic Advances in Drug Safety, 10, 2042098618820210.

Juthani-Mehta, M., Malani, P. N., \& Mitchell, S. L. 2015. Antimicrobials at the end of life: an opportunity to improve palliative care and infection management. Jama, 314(19), 2017-2018.

Kroschinsky, F., Stölzel, F., von Bonin, S., Beutel, G., Kochanek, M., Kiehl, M., \& Schellongowski, P. 2017. New drugs, new toxicities: severe side effects of modern targeted and immunotherapy of cancer and their management. Critical Care, 21(1), 89.

Lee, S. F. 2018. Antibiotics in palliative care: less can be more. Recognising overuse is easy. The real challenge is judicious prescribing. BMJ Support Palliat Care, 8(2):187-188.

Leonardi, G. C., Falzone, L., Salemi, R., Zanghì, A., Spandidos, D. A., Mccubrey, J. A., ... Libra, M. 2018. Cutaneous melanoma: From pathogenesis to therapy. International journal of oncology, 52(4), 1071-1080.

Levin, P. D., Simor, A. E., Moses, A. E., \& Sprung, C. L. (2010). End-of-life treatment and bacterial antibiotic resistance: a potential association. Chest, 138(3), 588-594.

Lima, C. 2006. Medicina High Tech, obstinação terapêutica e distanasia. Medicina Interna, 13(2), 79-82.

Macedo, F., \& Bonito, N. (2019). Current opinion about antimicrobial therapy in palliative care: an update. Current Opinion in Oncology, 31(4), 299-301.

Macedo, F., Nunes, C., Ladeira, K., Pinho, F., Saraiva, N., Bonito, N., ... Gonçalves, F. 2018. Antimicrobial therapy in palliative care: an overview. Supportive Care in Cancer, 26(5), 1361-1367.

Machado, K. K., Katz, A., Buyse, M., \& Saad, E. D. 2010. Sobrevida global e outros desfechos clínicos em câncer de mama: situação atual e controvérsias. Revista da Associação Médica Brasileira, 56(5), 514-516.

Merel, S. E., Meier, C. A., McKinney, C. M., \& Pottinger, P. S. 2016. Antimicrobial use in patients on a comfort care protocol: a retrospective cohort study. Journal of Palliative Medicine, 19(11), 1210-1214. 
Mirhosseini, M., Oneschuk, D., Hunter, B., Hanson, J., Quan, H., \& Amigo, P. 2006. The role of antibiotics in the management of infection-related symptoms in advanced cancer patients. Journal of Palliative Care, 22(2), 69-74.

Mohammed, A. A., Al-Zahrani, A. S., Sherisher, M. A., Alnagar, A. A., Ayman, E. S., \& El-Kashif, A. T. 2014. The pattern of infection and antibiotics use in terminal cancer patients. Journal of the Egyptian National Cancer Institute, 26(3), 147-152.

Nakagawa, S., Toya, Y., Okamoto, Y., Tsuneto, S., Goya, S., Tanimukai, H., ... \& Takagi, T. 2010. Can antiinfective drugs improve the infection-related symptoms of patients with cancer during the terminal stages of their lives?. Journal of Palliative Medicine, 13(5), 535-540.

Pereira, J., Watanabe, S., \& Wolch, G. 1998. A retrospective review of the frequency of infections and patterns Mof antibiotic utilization on a palliative care unit. Journal of Pain and Symptom management, 16(6), 374-381.

Prabhavathi F., \& Evan, M. 2017. Antibiotics in late clinical development. Biochemical Pharmacology, 133, 152-163.

Ramadas, L., \& Feijó Barroso, P. 2017. High frequency of antimicrobials use in palliative care: are we moving in the right direction?. Journal of Palliative Medicine, 20(3), 218-219.

Rosenberg, J. H., Albrecht, J. S., Fromme, E. K., Noble, B. N., McGregor, J. C., Comer, A. C., \& Furuno, J. P. 2013. Antimicrobial use for symptom management in patients receiving hospice and palliative care: a systematic review. Journal of Palliative Medicine, 16(12), 1568-1574.

Sarfati, D., Koczwara, B., \& Jackson, C. (2016). The impact of comorbidity on cancer and its treatment. CA: $a$ Cancer Journal for Clinicians, 66(4), 337-350.

Stiel, S., Krumm, N., Pestinger, M., Lindena, G., Nauck, F., Ostgathe, C., ... \& Elsner, F. 2012. Antibiotics in palliative medicine-results from a prospective epidemiological investigation from the HOPE survey. Supportive Care in Cancer, 20(2), 325-333.

Thai, V., Lau, F., Wolch, G., Yang, J., Quan, H., \& Fassbender, K. 2012. Impact of infections on the survival of hospitalized advanced cancer patients. Journal of Pain and Symptom Management, 43(3), 549-557.

Thompson, A. J., Silveira, M. J., Vitale, C. A., \& Malani, P. N. 2012. Antimicrobial use at the end of life among hospitalized patients with advanced cancer. American Journal of Hospice and Palliative Medicine ${ }^{\circledR}, 29(8)$, 599-603.

Vitetta, L., Kenner, D., \& Sali, A. 2000. Bacterial infections in terminally ill hospice patients. Journal of Pain and Symptom Management, 20(5), 326-334.

White, P. H., Kuhlenschmidt, H. L., Vancura, B. G., \& Navari, R. M. 2003. Antimicrobial use in patients with advanced cancer receiving hospice care. Journal of Pain and Symptom Management, 25(5), 438-443.

World Health Organization [WHO]. 2002. The Importance of Pharmacovigilance. Safety Monitoring of medicinal products. Geneva, $\mathrm{CH}$ : WHO.

Yılmaz, Ç., \& Özcengiz, G. (2017). Antibiotics: pharmacokinetics, toxicity, resistance and multidrug efflux pumps. Biochemical Pharmacology, 133, 43-62.

Zwaan, C. M., Kolb, E. A., Reinhardt, D., Abrahamsson, J., Adachi, S., Aplenc, R., ... \& Hasle, H. 2015. Collaborative efforts driving progress in pediatric acute myeloid leukemia. Journal of Clinical Oncology, 33(27), 2949.

\section{Minicurrículo}

Claudinei Alves Santana. Possui graduação em Farmácia Bioquímica e Homeopatia pela Universidade São Judas Tadeu (2004). Especialização em Farmácia Hospitalar pela Faculdade Oswaldo Cruz (2005) e Planejamento Educacional e Docência do Ensino Superior pela ESAB (2010). Especialização Multiprofissional em Oncologia pelo Hospital Sírio Libanês (2019). Mestre em Ciências Médicas pela Faculdade de Medicina da USP (2014). Farmacêutico responsável pelo Serviço de Extensão ao Atendimento de Pacientes HIV/AIDS do HC-FMUSP (2005 -2013). Docente da Pós Graduação SENAC/SP. Docente da Pós Graduação ICTQ. Experiência em Manipulação, Acompanhamento clínico e Dispensação de Quimioterápicos. Experiência em Farmácia Clínica, Ensino, Assistência a Pacientes HIV/AIDS. 
Mirian de Freitas Dal Ben Corradi. Possui graduação em Medicina pela Universidade Federal de Minas Gerais (2003), residência médica pela Universidade de São Paulo (2007). Mestrado e Doutorado pelo Departamento de Doenças Infecciosas e Parasitárias da Faculdade de Medicina da USP. Tem experiência na área de Medicina, com ênfase em Doenças Infecciosas e Parasitárias e Infecção Hospitalar. Atualmente é médica assistente do Serviço de Controle de Infecção Hospitalar do Hospital Sírio Libanês. É membro da diretoria da Associação Brasileira de Infecção Hospitalar e foi membro da Comissão Científica da Associação Paulista de Epidemiologia e Controle de Infecção Relacionada à Assistência à Saúde (2011-2014).

Silvia de Lima Vieira. Graduação em Enfermagem pela Universidade de Guarulhos (UNG) e Mestrado em Enfermagem na Saúde do Adulto pela Universidade de São Paulo (EEUSP). Especialização em Administração Hospitalar, Unidade terapia Intensiva e Segurança do Paciente. Gerente de enfermagem do Hospital Sírio Libanês, Coordenadora do Programa de Residência Multiprofissional em Oncologia e do Curso de Especialização Multiprofissional em Oncologia do Hospital Sírio Libanês. Consultora no Processo de Qualidade e Segurança do Paciente do Hospital e Maternidade São Lucas Extrema/ MG.

Wanessa Cassemiro Fernandes. Doutoranda no Programa de Pós-Graduação Stricto Sensu em Ciências da Saúde do Instituto Sírio Libanês de Ensino e Pesquisa. Mestre em Enfermagem pela Universidade de São Paulo (2009), especialização Lato Sensu em Enfermagem Oncológica pelo Centro Universitário São Camilo (2006), graduação em Bacharel em Enfermagem pela Universidade Cidade de São Paulo (2000). Atualmente é enfermeira Sênior na Unidade de Internação Oncológica do Hospital Sírio-Libanês; Docente/tutora no Programa de Residência Multiprofissional em Saúde no Cuidado ao Paciente Oncológico e Coordenadora do Curso de Pós Graduação Lato Sensu Multiprofissional em Oncologia do Instituto Sírio Libanês de Ensino e Pesquisa.

Como citar: Santana, C.A., Corradi, M.F.D.B., Vieira, S.L., \& Fernandes, W.C. 2020. Uso de antimicrobianos em pacientes oncológicos em cuidados paliativos. Pubsaúde, 3, a058. DOI: https://dx.doi.org/10.31533/pubsaude3.a058

Recebido: 29 jul. 2020

Revisado e aceito: 2 ago. 2020.

Conflito de interesse: os autores declaram, em relação aos produtos e companhias descritos nesse artigo, não ter interesses associativos, comerciais, de propriedade ou financeiros que representem conflito de interesse.

Licenciamento: Este artigo é publicado na modalidade Acesso Aberto sob a licença Creative Commons Atribuição 4.0 (CC-BY 4.0). 